$G$

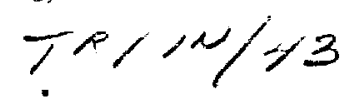

NAG/-1927

\title{
USING GPS REFLECTIONS FOR SATELLITE REMOTE SENSING
}

\author{
David Mickler, George Born \\ Colorado Center for Astrodynamics Research, Boulder, CO
}

GPS signals that have reflected off of the ocean's surface have shown potential for use in oceanographic and atmospheric studies. The research described here investigates the possible deployment of a GPS reflection receiver onboard a remote sensing satellite in low Earth orbit (LEO). The coverage and resolution characteristics of this receiver are calculated and estimated. This mission analysis examines using reflected GPS signals for several remote sensing missions. These include measurement of the total electron content in the ionosphere, sea surface height, and ocean wind speed and direction. Also discussed is the potential test deployment of such a GPS receiver on the space shuttle. Constellations of satellites are proposed to provide adequate spatial and temporal resolution for the aforementioned remote sensing missions. These results provide a starting point for research into the feasibility of augmenting or replacing existing remote sensing satellites with spaceborne GPS reflection-detecting receivers.

\section{INTRODUCTION}

The Global Positioning System (GPS) is finding new applications with increasing frequency. One of the most recent innovations includes using GPS bistatic signal scattering for ocean remote sensing. A high altitude GPS receiver, properly initialized and calibrated, is used to track GPS signals that have reflected off of the ocean's surface. This receiver is used in conjunction with a Left Hand Circularly Polarizing (LHCP) antenna, since the polarity of the GPS signal reverses upon reflection. This research investigates several low Earth orbiting (LEO) satellite missions that would enable this new technology to provide inexpensive global ocean remote sensing.

First we will discuss the emerging field of GPS reflection measurement. Then we will discuss some possible uses for this field. These remote sensing missions could be implemented in several ways. Here we propose LEO satellite constellations to provide the necessary spatial and temporal sampling characteristics. We also investigate mounting a prototype reflection receiver on the space shuttle. Such an experiment would help verify the techniques and models being developed will work from LEO before mounting a dedicated reflection receiver on a scientific satellite. The distribution, azimuth, and elevation angle of the reflected GPS signals as seen by the space shuttle are calculated through simulation and evaluated. 
The background material first covers bistatic signal scattering of GPS signals and their subsequent measurement. Then we discuss some of the literature and necessary . astrodynamics needed for constellation design. The coverage and resolution characteristics of the potential GPS bounce-measuring instrument are also discussed.

\section{GPS Bounce Concept}

The first to propose using reflected GPS signals for oceanography was Martin-Neira ${ }^{\mathrm{I}}$. Then, in 1994, French engineers reported the accidental acquisition of ocean reflected GPS signals by an aircraft mounted receiver. This study was documented by Auber et al ${ }^{2}$. Katzberg and Garrison ${ }^{3}$ recognized the potential for this new remote sensing technique. Results from the application of their theories are found in Garrison et $\mathrm{al}^{4}$. The work done to modify a GPS receiver into a reflected signal detector that basically measures multipath is detailed by Garrison et al ${ }^{5}$.

Such an instrument could conceivably be used for several oceanographic applications. Martin-Neira ${ }^{\prime}$ discussed the potential for reflected GPS signals to perform altimetry. Katzberg and Garrison ${ }^{3}$ provide the technique to calculate ionospheric delay from the dual frequency backscattered signals. Komjathy et al. ${ }^{6}$ describe how the signal scattering could be used to perform scatterometry with a properly configured receiver. We will conduct a mission analysis to determine the feasibility and characteristics of using such a properly equipped receiver on a LEO remote sensing satellite mission. The ability of a GPS receiver to perform each of these missions, altimetry, scatterometry, and ionospheric measurement, will be examined in terms of spatial and temporal resolution.

\section{Possible Bounce Missions}

There are several applications of knowing the current state of the ionosphere. The ionospheric delay is one of the largest sources of error for GPS Wide Area Augmentation Systems (WAAS) (see Komjathy ${ }^{7}$ ). Having an ionosphere-measuring constellation would provide a realtime correction for many remote sensing instruments and GPS users. In order to provide a useful measurement of the ionosphere (such as a real-time correction for scientific satellites), in particular the Total Electron Content (TEC), the instrument would have to achieve a certain resolution. This resolution needs to be on the same scale as the spatial decorrelation length and decorrelation time of the TEC. To provide a useful global map of the ionosphere one needs to achieve a resolution of roughly $500 \mathrm{~km}$ spatially and 2 hours temporally. Obviously the only way to achieve such high temporal resolution is by deploying a constellation of remote sensing satellites. One may suggest adding instruments to a geostationary satellite but the reflected GPS signals would most likely be too weak to be measured from beyond LEO. The TEC, a measure of ionospheric activity, is measured by differencing the two carrier frequencies (1575.42 and 1227.60 $\mathrm{MHz}$ ) that GPS is currently transmitted on. Currently, no global ionospheric measurements have been taken in real time. The International GPS Service (IGS) provides dual frequency data that can be used to form global TEC maps after processing (see Komjathy ${ }^{7}$ ). 
Several scatterometry missions have flown or are manifested. NSCAT, which flew on a short-lived mission, and QuikScat, were designed to measure global ocean wind speed and direction. The scatterometers achieve global coverage in 2 days with $50 \mathrm{~km}$ spatial resolution. The QuikScat instrument has a $1,800 \mathrm{~km}$ swath width and covers $90 \%$ of the ocean's surface in a day from its $803 \mathrm{~km}$ sun-synchronous orbit. The stated wind vector resolution of the instrument is $25 \mathrm{~km}$. The accuracy in determining wind speed and direction is $2 \mathrm{~m} / \mathrm{sec}$ (in a range from 3 to $20 \mathrm{~m} / \mathrm{sec}$ ) and $20 \mathrm{deg}$, respectively. This work attempts to make a comparison with the wind vector resolution.

Altimetry has a longer heritage. The most accurate altimeter currently in use is TOPEX/Poseidon, which achieves $2 \mathrm{~cm}$ RMS accuracy in its measurements, which are repeated every 10 days. The ERS-2 altimeter also currently produces ocean topographic maps every 35 days at a reduced accuracy of about $17 \mathrm{~cm}$. The high level of accuracy achieved by TOPEX is gained mainly through advances in orbit determination. If a GPS reflection receiver were mounted on the TOPEX follow-on mission (JASON) or a future generation of altimeter this same accuracy could be taken advantage of. Real-time observed ionospheric corrections could also be obtained. Currently altimetry missions use a model-derived ionospheric correction.

\section{LEO Constellations}

Using constellations of satellites decreases observation repeat times. The more satellites in a uniform constellation the more often a point on the Earth's surface is under observation, and the shorter the time gaps when that point is not under observation. Global coverage cannot be achieved from LEO by a single satellite in much less than a day because the instruments onboard cannot view a wide enough area of the Earth's surface. LEO is here considered to be about $1000 \mathrm{~km}$ or less, within the range of most small launchers in operation today. The coverage angle geometry of a satellite in orbit is related to the altitude of the spacecraft above the Earth's surface and the instrument's field of view. The geometry is diagrammed in Figure 1. The instrument field of view is the angle $h$. The angular radius of the Earth, which is the cone-angle of the Earth's surface visible from a certain altitude $h$, is labeled 1 . See Wert $z^{8}$ for a full exposition on coverage angles. For a GPS bounce receiver we can consider $\eta=\rho$.

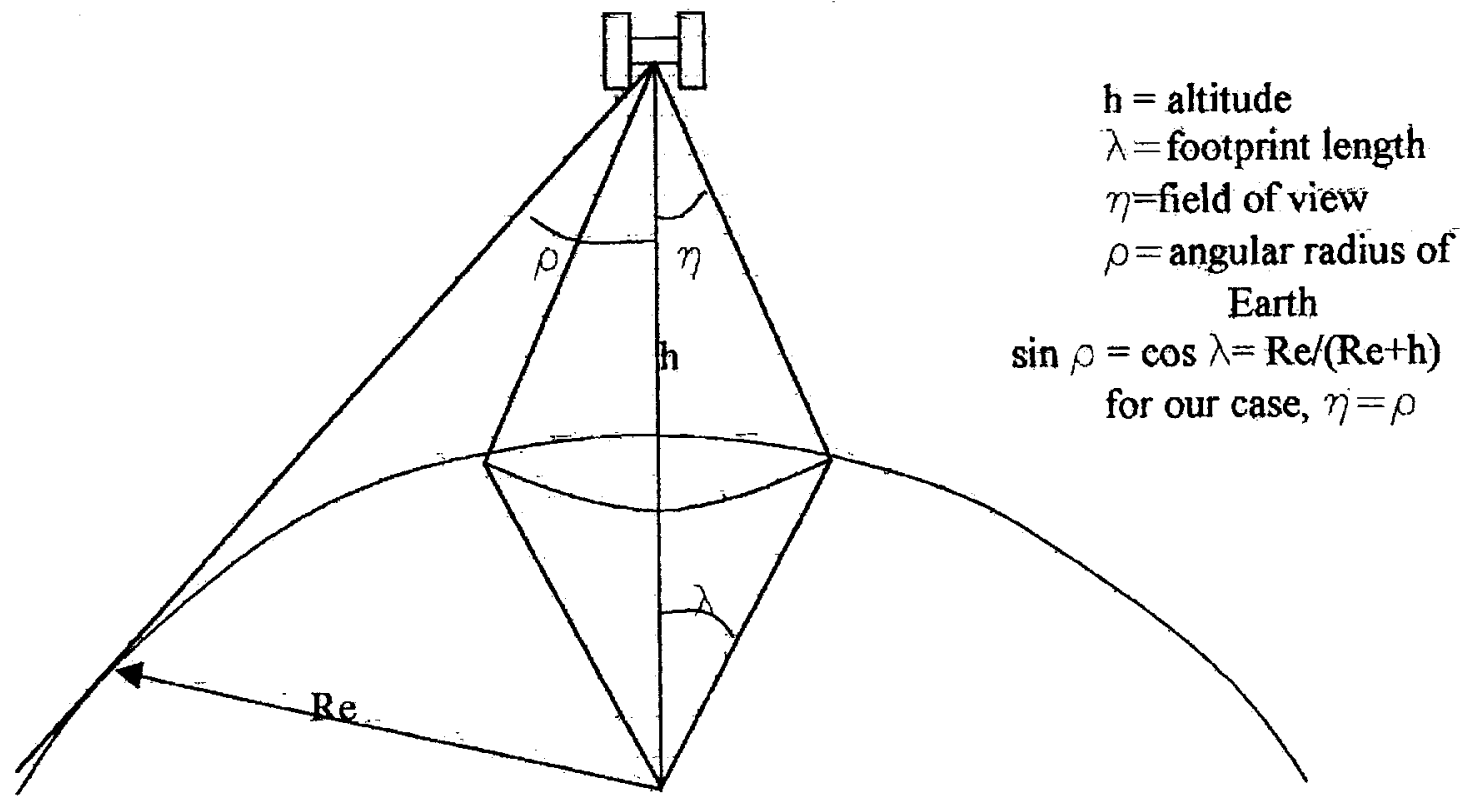

Figure I - Nadir Pointing Instrument Viewing Angle Gcometry 
Two orbital elements affect the cost of placing a satellite in orbit, and hence the feasibility of a new remote sensing technology in an era of limited budgets. The altitude of the orbit desired is the main factor affecting how much a launch costs. A certain launch vehicle can place a satellite of a specified mass within a certain orbit. With a larger launch vehicle the spacecraft mass or altitude can be increased, or a mixture of both. The smallest, and therefore least expensive, launchers are intended for spacecraft of less than 1 metric ton and for altitudes of $800 \mathrm{~km}$ or less. The altitude needs to be high enough to mitigate the effects of atmospheric drag and allow a large instrument viewing footprint. The altitude (of a circular orbit) needs to be above $200 \mathrm{~km}$ to ensure a mission lifetime of one year. However, in most cases, the lower the altitude the better the resolution of the instrument.

The other orbital element increasing the launch cost is inclination. A satellite's minimum inclination is limited by the latitude of the launchpad. This can be seen from the equation for orbit inclination $i, \cos (i)=\cos \left(\Phi_{\mathrm{gc}}\right) \sin (\beta)$, where $\Phi_{\mathrm{gc}}$ is the geocentric latitude of the launch site, and $\beta$ is the launch azimuth of the rocket (from Vallado ${ }^{9}$ ). The more that $\beta$ deviates from the direction of Earth's velocity the higher the velocity the launcher needs to impart to get the satellite to its desired orbit. Thus, to achieve higher inclinations than the launch latitude, it is necessary to burn more fuel for the same orbital altitude. The higher the inclination the larger the zone of coverage a satellite can achieve. A polar orbiting spacecraft can view every latitude of the Earth.

Thus a constellation of satellites can be used to achieve multiple coverage planes. A constellation plan attempts to optimize by selecting the fewest number of satellites, the lowest inclination, and the lowest altitude. The objective function to be optimized finds the orbital elements needed to achieve the desired coverage subject to the minimizing constraints.

Much work has been done on satellite constellation patterns. The first paper on constellations was by $\operatorname{Vargo}^{10}$. Most of the work since that time has been on continuous global coverage from altitudes above LEO. The seminal works are by Walker" Draim $^{12,13}$, Lang $^{14,15}$, and Ballard ${ }^{16}$. One of the first works on zonal coverage was by Rider ${ }^{17}$. For our case global coverage is not needed. Ocean scatterometry and altimetry are not as useful over the polar regions, unless to detect the edge of the pack ice. Research is underway regarding GPS reflections from ice, but results have yet to be published. Lang and Hanson ${ }^{18}$ discuss minimizing revisit times. That is to say, how soon does a certain constellation provide repeat coverage of a certain location? After the location has been observed, how much time passes until the location comes under observation again? A short revisit time is needed to meet our temporal resolution requirements, especially for an ionospheric mapping mission. Another good treatise on low altitude constellation design is by Hanson and Linden ${ }^{19}$. Hanson et al.' $\mathrm{s}^{20}$ paper on partial coverage satellite constellations was the most applicable to this work and is used extensively in these results. They define 'best' to mean a minimum number of satellites at a minimum possible inclination with the smallest maximum, or shortest, revisit time. These criteria are the same needed by a small satellite mission such as the one proposed here. 


\section{METHODS}

First we needed to estimate the spatial resolution of a GPS reflection tracking receiver. The area observed by a satellite is called the footprint. The smallest detail that can be discerned within the footprint is typically called the spatial resolution. In this case, the amount of the footprint that is sampled is also related to the resolution. The reflection measuring instrument is nominally nadir pointing to maximize the gain from all areas of its footprint along the groundtrack. Within the instrument's field of view, the signals from several GPS satellites will be reflecting at spectral points. The location of the bounce points can be solved for using an algorithm from Wu et al. ${ }^{21}$ and Armatys ${ }^{22}$. The size of these specular reflection areas determines what area of the ocean's surface is sampled. The size of the specular reflection area is determined by the ocean surface's wind speed, which determines surface roughness. Also, the higher the altitude of the observing instrument, the larger the glistening zone. These reflection areas are well illustrated in Figure 2 of Komjathy et al. ${ }^{6}$

A simulation was set up for this research that calculated the reflection points between the GPS constellation and a sample reflection receiver in LEO at $350 \mathrm{~km}$ altitude. A receiver mask was used to eliminate any GPS satellites whose reflection had traveled through too much of the ionosphere. This precluded the receiver from using any reflections with an incidence angle $\alpha$ greater than $70 \mathrm{deg}$. There was an average of 9 GPS satellite reflection points available for tracking at any one time. This number was as low as 6 and as high as 12. The average specular point diameter, labeled $d$ in Figure 2, is 65 $\mathrm{km}$. This corresponds to an average wind speed of 5 to $8 \mathrm{~m} / \mathrm{sec}$, as seen from a $350 \mathrm{~km}$ altitude. This yields an average specular reflection area of just over $3,000 \mathrm{~km}^{2}$. The field of view $\eta$, calculated from the equations in Figure 1, is estimated to be $70 \mathrm{deg}$. This corresponds to a swath width of $4102 \mathrm{~km}$ and a footprint area of $3,345,000 \mathrm{~km}^{2}$ of the Earth's surface. To find an estimate of the Earth's surface sampled by a reflection receiver we estimate the percentage of the area sampled by the specular reflection area as compared to the footprint area. This resolution concept is illustrated in Figure 2. We use this estimate of area of the footprint sampled along the groundtrack to estimate the spatial resolution of a GPS reflection receiver.

$$
\begin{aligned}
& \mathrm{Lf}=111.319 \mathrm{~km} / \mathrm{deg} \mathrm{I} \\
& \mathrm{Fa}=\text { footprint area } \\
& \mathrm{Fa}=(\mathrm{p} / 4) \mathrm{Lf}^{2}
\end{aligned}
$$

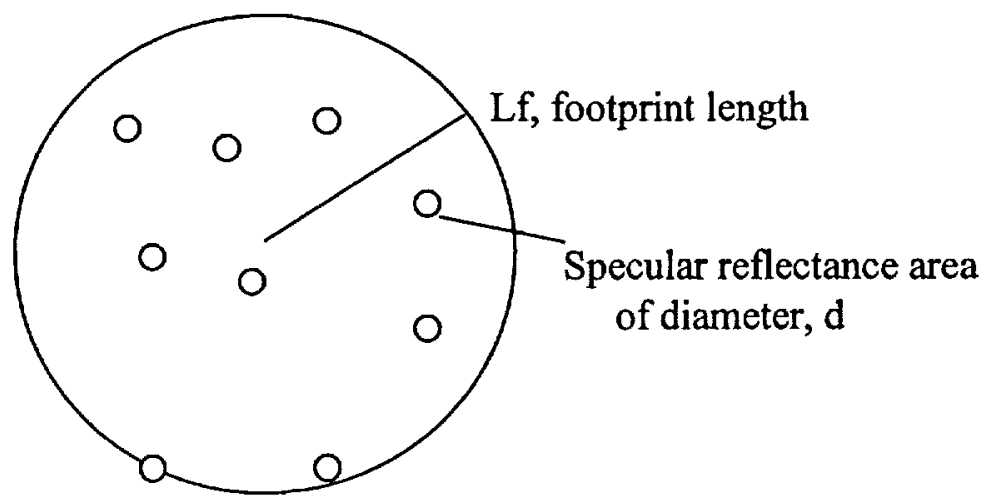

Figure 2 - Resolution Estimate 
For the viewing angle study the space shuttle's orbit parameters are used in the simulation. We look here at two classes of shuttle missions, split by inclination. The basic shuttle science mission has an inclination of $28 \mathrm{deg}$, whereas an International Space Station (ISS) service mission currently has an inclination of $52 \mathrm{deg}$. From the simulation calculations we can observe several parameters that potentially affect the bounce receiver design and mounting. These include the azimuth and elevation angles to the reflection. The azimuth angle measures the orientation of the GPS constellation with respect to the shuttle's inertial velocity direction. The elevation angle measures how low the GPS satellite is on the shuttle's horizon. The larger the elevation angle, the lower the signal to noise ratio the bounce receiver sees. The elevation angle $\alpha$, which is measured here from the nadir direction, is illustrated in Figure 3.

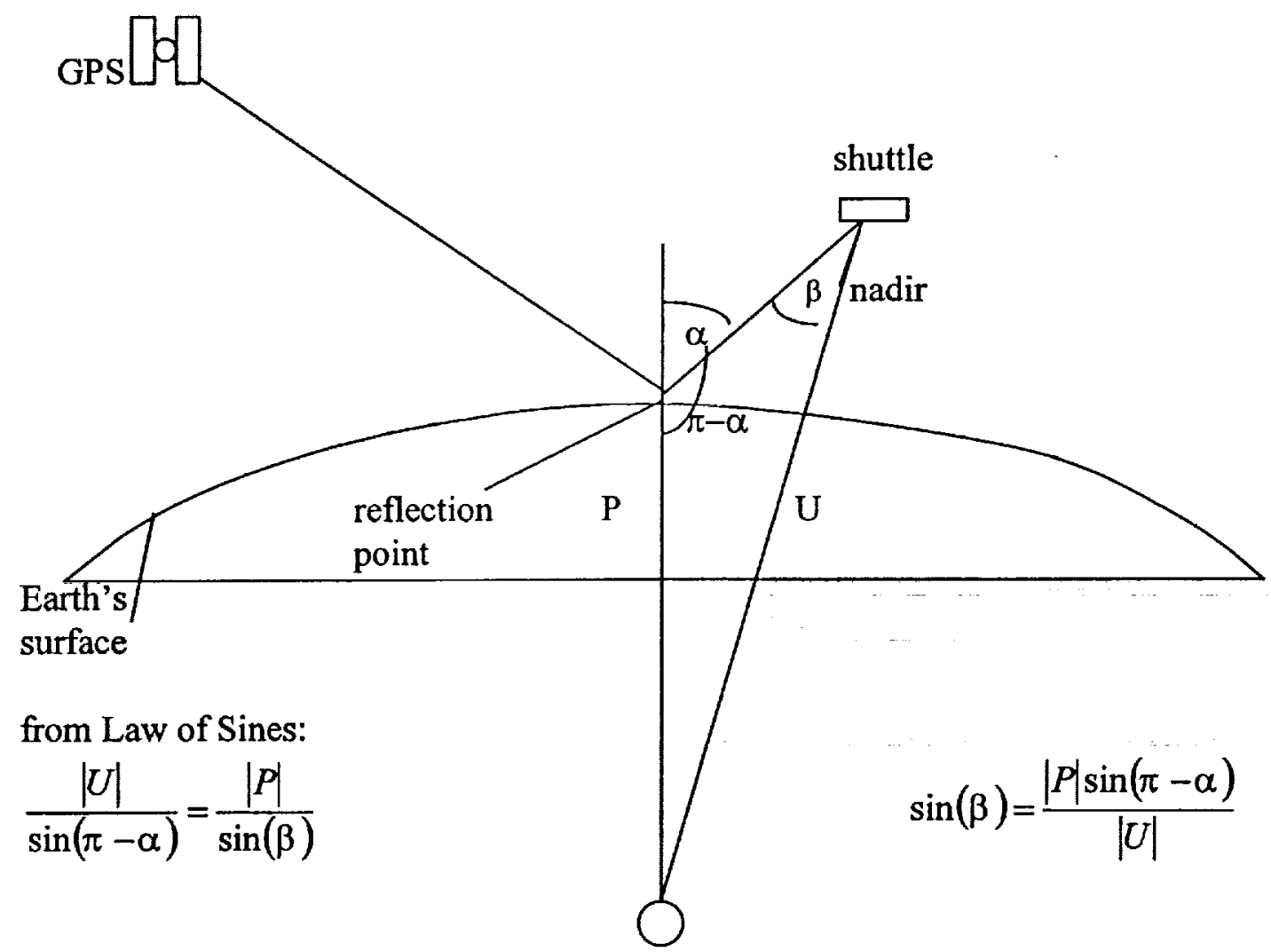

center of ECEF frame

Figure 3-Geometry of the reflected signal

Coverage calculations were computed using the grid method. A grid of coverage was defined between the latitude bounds of the study region. For altimetry and 
scatterometry, we adopt the same bounds as TOPEX and QuikScat, roughly 70 deg. That is, we measure the coverage ability of our constellation designs between -70 and 70 deg $\mathrm{N}$ latitude. For ionospheric mapping we use a grid with $500 \mathrm{~km}$ spatial resolution. For scatterometry we use a grid with $50 \mathrm{~km}$ spatial resolution, emulating that of existing missions. Our simulation divides the globe into squares with area equivalent to the desired spatial resolution, either 500 or $50 \mathrm{~km}$. Then the satellite groundtracks and coverage swaths are projected over these grids. We consider full coverage to be when a satellite views all the grids within the desired temporal resolution. Percent coverage is calculated based on what percent of the grid is observed by the satellite in a specified time. Repeat time analysis is done by calculating how long a certain grid area goes unobserved.

Altimetry missions are a little different because the altimeter samples in a narrow band directly beneath its groundtrack. Thus the main difference between a radar altimeter and a reflection receiver is that an altimeter has one instrument groundtrack that repeats and a GPS reflection receiver has an average of 9 non-repeating groundtracks. Since spatial resolution is not an issue, we did not compute grid coverage simulations for altimetry missions.

\section{RESULTS}

\section{Viewing Angle Study}

The first results presented here are from the viewing angle study involving the space shuttle. Figure 4 shows the location of the specular reflection points seen from the space shuttle on a typical day, using the locations of the GPS constellation on March 30, 1998. A $1 / 4$-degree land mask was used to calculate these results. One can see that the GPS reflection points form multiple instrument groundtracks beneath the shuttle's groundtrack. This dataset is used to examine the azimuth and elevation angles seen by the reflection receiver.

Next we look at a histogram of azimuth angles for both shuttle missions, the low and the high inclination. For both missions the results in Figure 5 are basically the same. One can see that the azimuth angles are nearly uniformly distributed about the nadir pointing antenna of the reflection receiver. The azimuth angles were sampled at a period of once every 30 seconds. Thus each sample constitutes a point in the histogram.

The next figure examines the elevation angles of the GPS reflection points, as defined in Figure 3. A histogram of the elevation angles seen on March 30, 1998, is presented in Figure 6. One can see that elevations greater than 70 deg are masked out. This previously mentioned receiver mask is a standard procedure for a spaceborne GPS receiver. These elevation angles were also calculated at 30 second intervals. We see that the observing platform, here the space shuttle on a high inclination mission, is not often directly underneath a GPS satellite. This is mainly due to the fact that the shuttle, in this simulation, is not in the same orbital plane as the GPS spacecraft. When a GPS satellite is nearly overhead of the observing platform the elevation angle is less than $10 \mathrm{deg}$. This 
occurs less than $3 \%$ of the time for a typical day, according to Figure 6 . The elevation angle is greater than 50 deg over half the time. Results from other days were similar to those shown here.

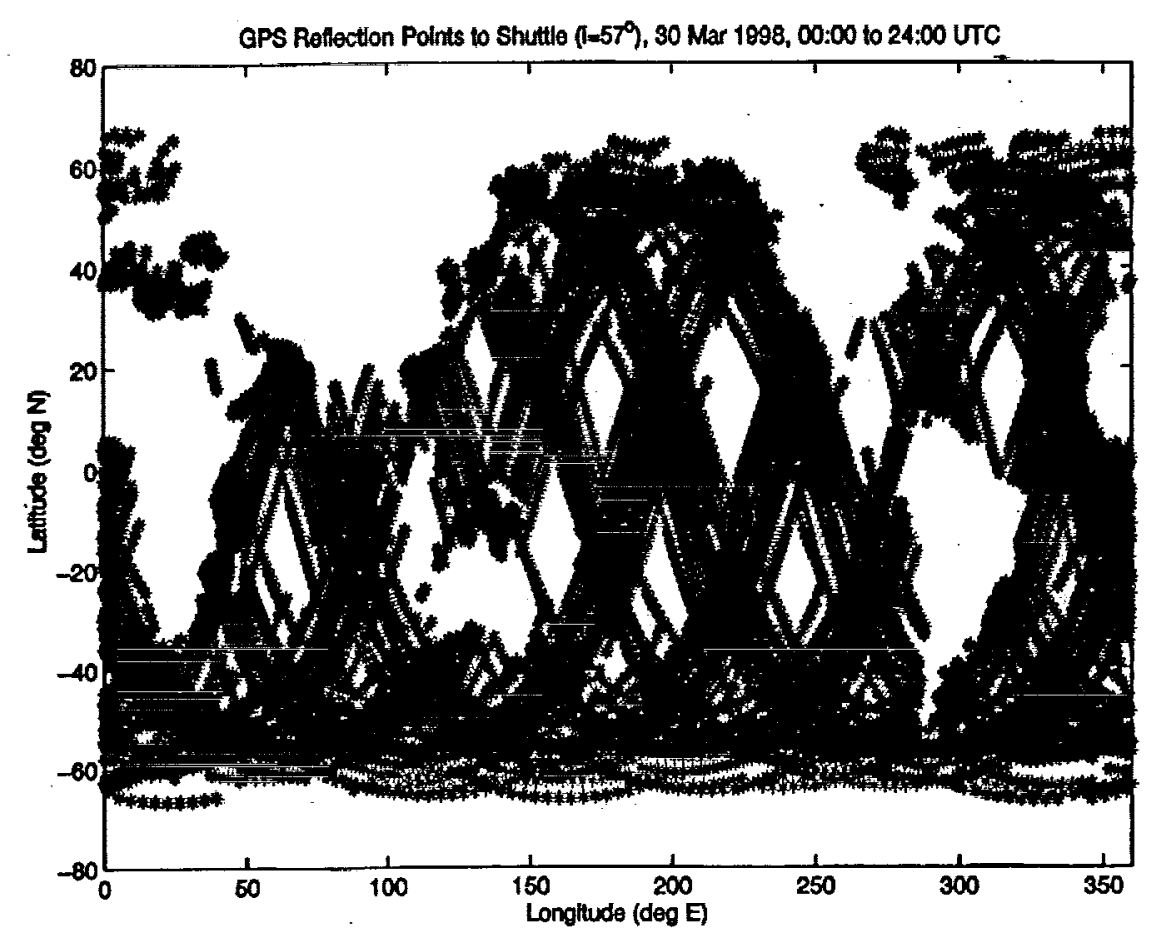

Figure 4 - Simulation of GPS Reflection Points as seen in High Inclination Orbit

Figure 7 shows a scatter plot of elevation angle versus latitude of the observing platform's groundtrack. One notices a bias toward higher elevation angles. One can also see a higher number of reflections over the southern ocean. This makes sense because similar latitudes in the northern ocean are dominated by the Asian and North American landmasses.

Several things can be said to conclude the viewing angle study. The antenna should be mounted pointing down, in the nadir direction, to avoid biasing reflections from one side or the other. This conclusion can be drawn from Figure 5 and the uniformity of azimuth angle distribution. The predominance of low elevation angles leads to the suggestion that the antenna gain pattern be designed such as to maximize the signal gain from lower elevations.

\section{Constellation Design}

A constellation was selected to perform the mission of ionospheric mapping in real time. The selection was made by minimizing the number of satellites needed and the inclination of their orbital planes. The orbital groundtracks during the 2-hour temporal resolution time period are shown in Figure 8. The constellation consists of four spacecraft 
in four separate orbital planes. The planes are at two different orbital inclinations.

Stationkeeping is minimal because the orbits selected are repeat groundtrack orbits (from Hanson et al. ${ }^{20}$ ). The Keplerian orbital elements for each plane are presented below in Table 1.
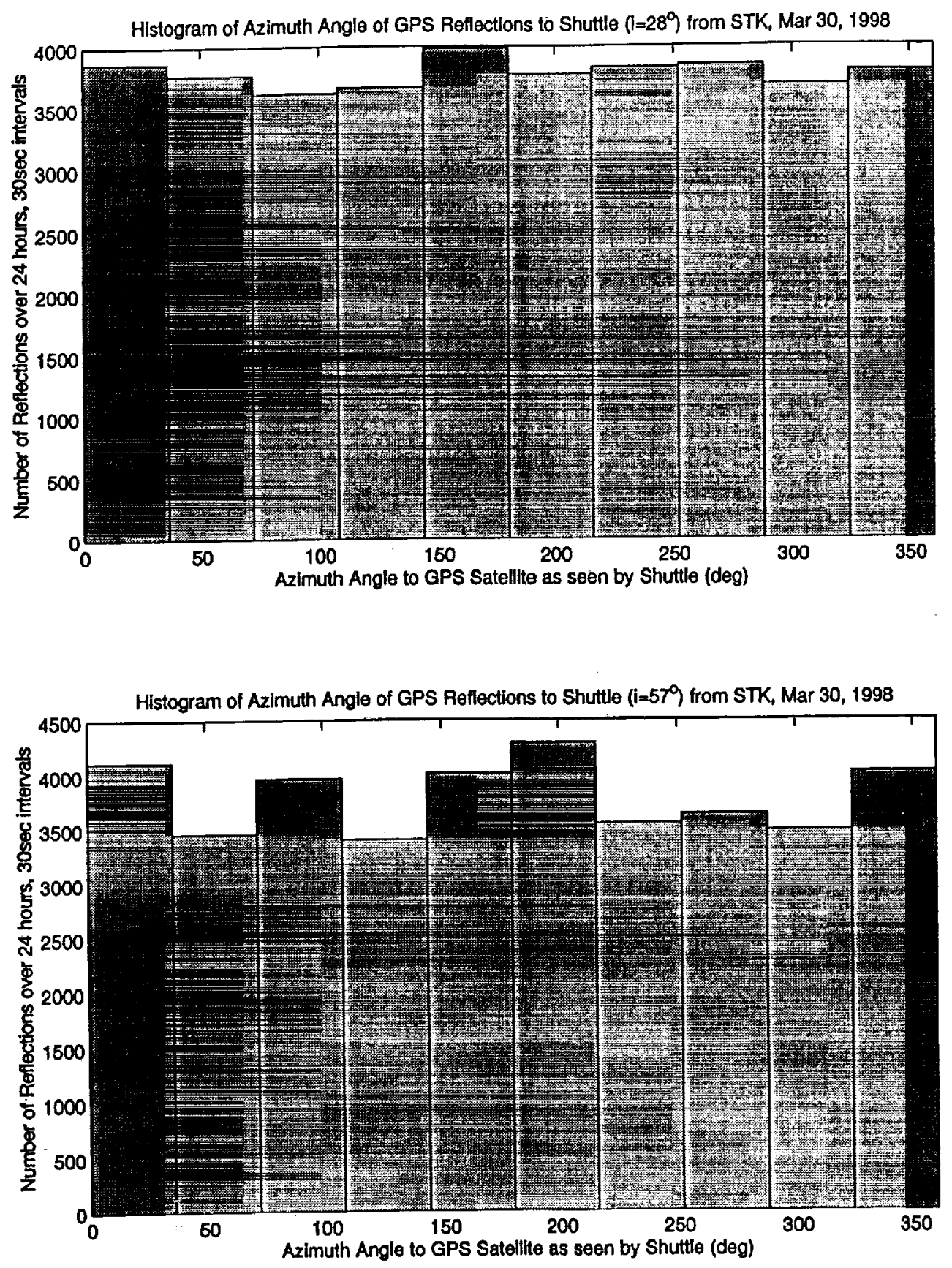

Figure 5 - Histograms of Azimuth Angle 


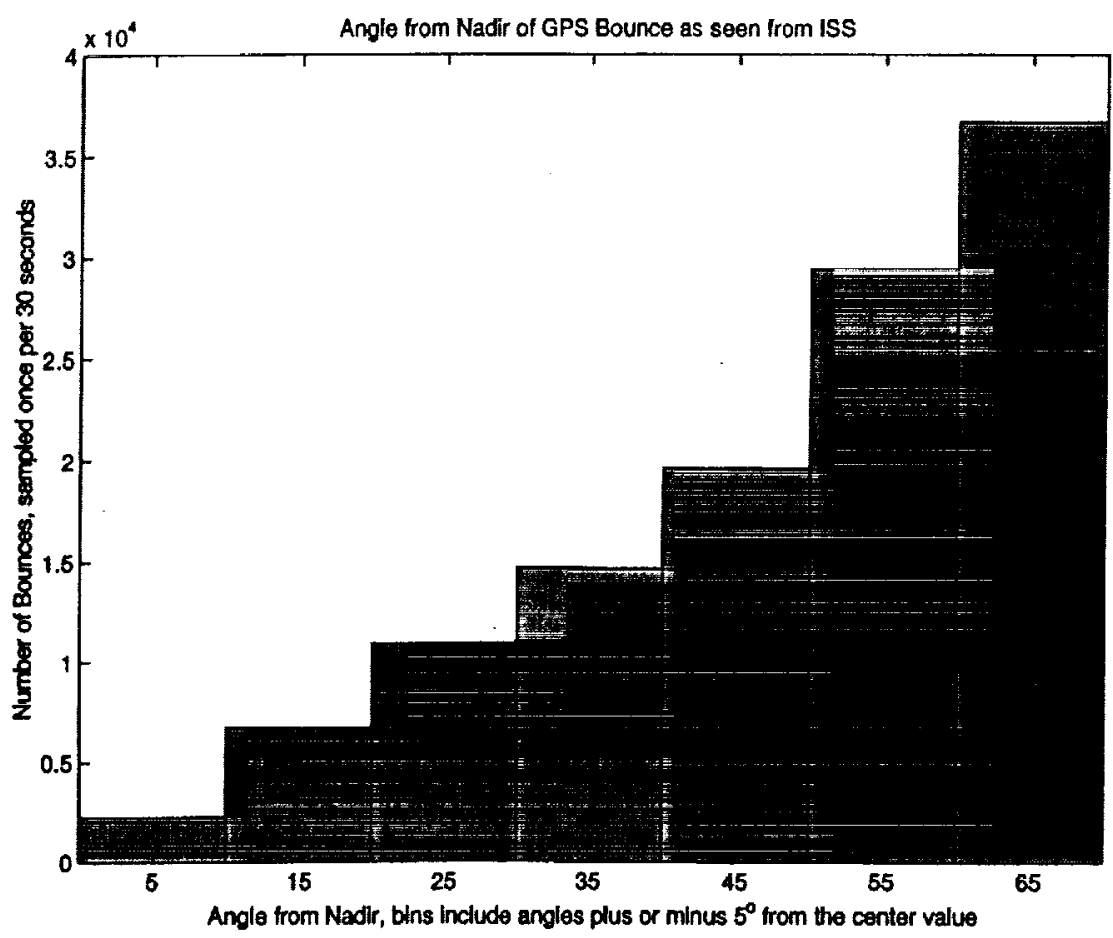

Figure 6 - Histogram of Angle from Nadir

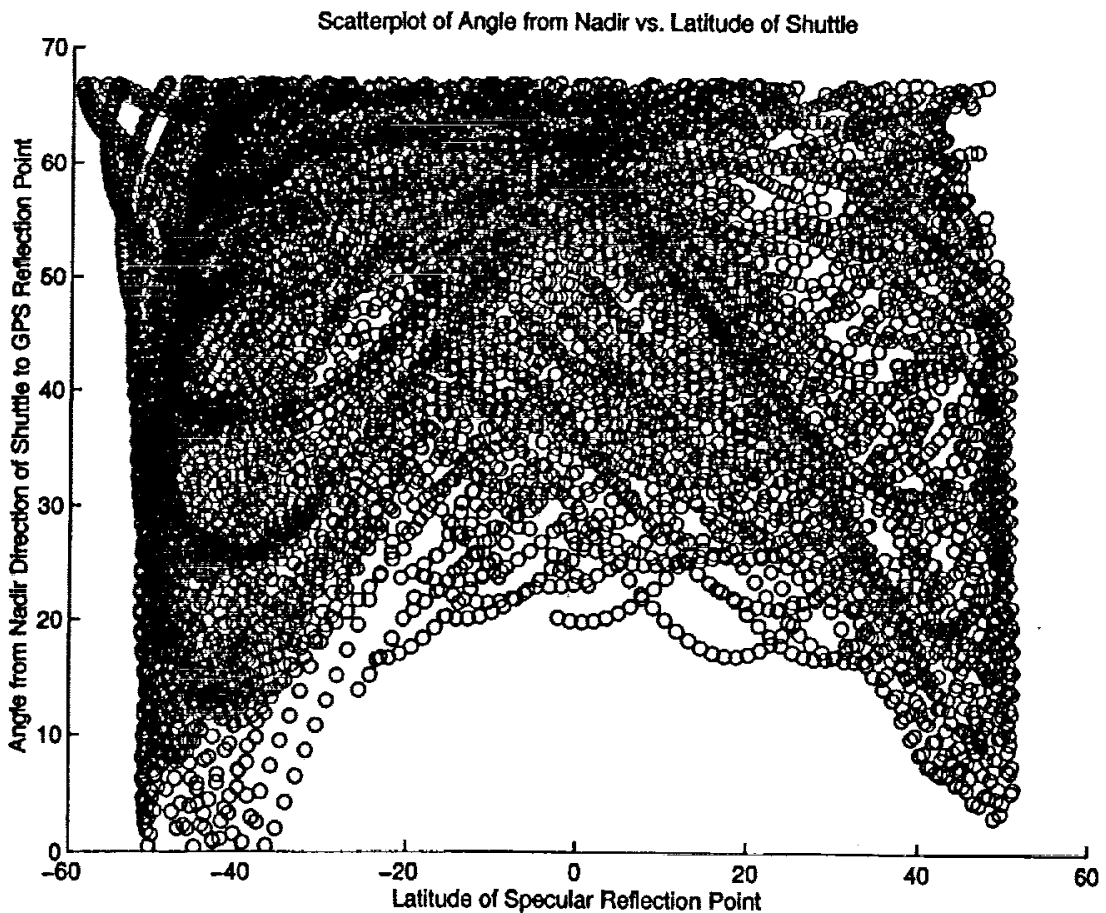

Figure 7 - Scatterplot of Angle from Nadir 
Table 1 - Keplerian Orbital Elements for Ionospheric Measurement Constellation

\begin{tabular}{|c|c|c|c|c|c|c|}
\hline Element & $\underline{a}(\mathrm{~km})$ & $\underline{\mathrm{e}}$ & i(deg) & $\underline{W}(\mathrm{deg})$ & $W(\operatorname{deg})$ & f(deg) \\
\hline High 1 & 6901.706 & 0 & 68 & 0 & $26 \overline{66.8662}$ & 0 \\
\hline High 2 & 6901.706 & 0 & 68 & 0 & 86.8662 & 180 \\
\hline Low 1 & 6862.636 & 0 & 29 & 0 & 86.8662 & 0 \\
\hline Low 2 & 6862.636 & 0 & 29 & 0 & 266.8662 & 180 \\
\hline
\end{tabular}

The orbital elements in Table 1 are as follows; $a$ is the semimajor axis in kilometers, $e$ is the eccentricity, $i$ is the inclination in degrees, $W$ is the argument of perigee in degrees, $W$ is the Right Ascension of the Ascending Node in degrees, and $f$ is the true anomaly in degrees. The two high inclination satellites, labeled High 1 and High 2 in Table 1, are at an altitude of $523.569 \mathrm{~km}$. The two low inclination spacecraft, labeled Low 1 and Low 2 in Table 1, are at an altitude of $484.499 \mathrm{~km}$. These orbits are repeating groundtrack orbits selected from Hanson et al..$^{20}$ They were selected to meet the desired temporal resolution of two hours. Coverage tests were run which concluded that the desired spatial resolution of $500 \mathrm{~km}$ was met $95 \%$ of the time. This corresponds to $95 \%$ coverage of the desired area. Total $(100 \%)$ coverage of the latitude bounds was satisfied with temporal resolution of about 11.8 hours. The repeat time is slightly higher than 11.8 hours for the high inclination planes and slightly lower for the low inclination planes.

For the scatterometry mission it was found that a single spacecraft could meet the required resolution. This indicates that a bounce receiver could even be mounted on a future scatterometry mission, providing a redundant independent measurement source. Such an uncorrelated independent measurement, capable of correcting the ionospheric delay, would certainly increase the accuracy of a scatterometer. The scatterometry orbit is also selected to have a repeating groundtrack. The groundtrack is shown in Figure 9. The repeat time for this orbit is $10.73 \mathrm{hr}$. The Keplerian orbital elements for the selected scatterometry mission are listed in Table 2 . The elements are the same as the ones described for Table 1 above.

Table 2- Keplerian Orbital Elements for Scatterometry Mission

$\begin{array}{lllllll}\text { Elements } & \frac{\mathrm{a}(\mathrm{km})}{6910.284} & \frac{\mathrm{e}}{0} & \frac{\mathrm{i}(\mathrm{deg})}{74} & \frac{\mathrm{W}(\mathrm{deg})}{0} & \frac{\mathrm{W}(\mathrm{deg})}{199.3549} & \frac{\mathrm{f}(\mathrm{deg})}{0}\end{array}$

The altitude of this orbit is $532.147 \mathrm{~km}$. The inclination allows coverage of the global oceans. The coverage calculations indicate that for this particular orbit $100 \%$ coverage at a $50 \mathrm{~km}$ resolution is obtained in $12 \mathrm{hr} 4 \mathrm{~min}$.

The coverage calculations quoted here were computed assuming the 70 deg field of view. However, as seen in Figure 2, the reflection points do not fill this footprint. Assuming that the reflection points to the GPS satellites are not repeated from day to day, a different part of the footprint is sampled after each pass. Thus over the requisite 2 day temporal resolution the $500 \mathrm{~km}$ spatial resolution should be met. This would require further research into appropriate interpolation techniques. One suggestion would be to use 
a weighted least squares routine for the region about each grid point and use that to get a fit for each grid point as well. However, our main concern here is feasibility.

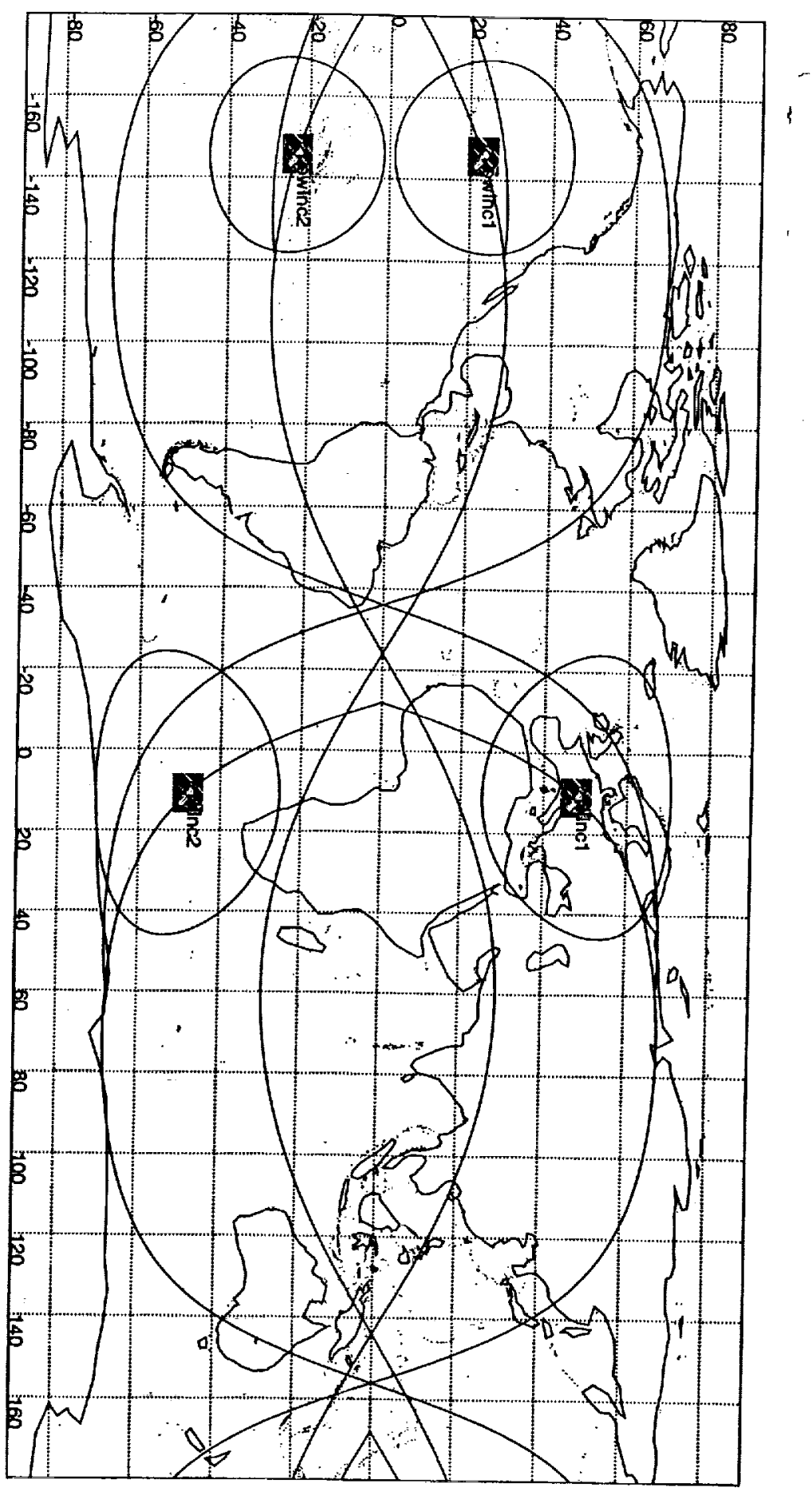

Figure 8 - Ionospheric Measurement Constellation Groundtracks 


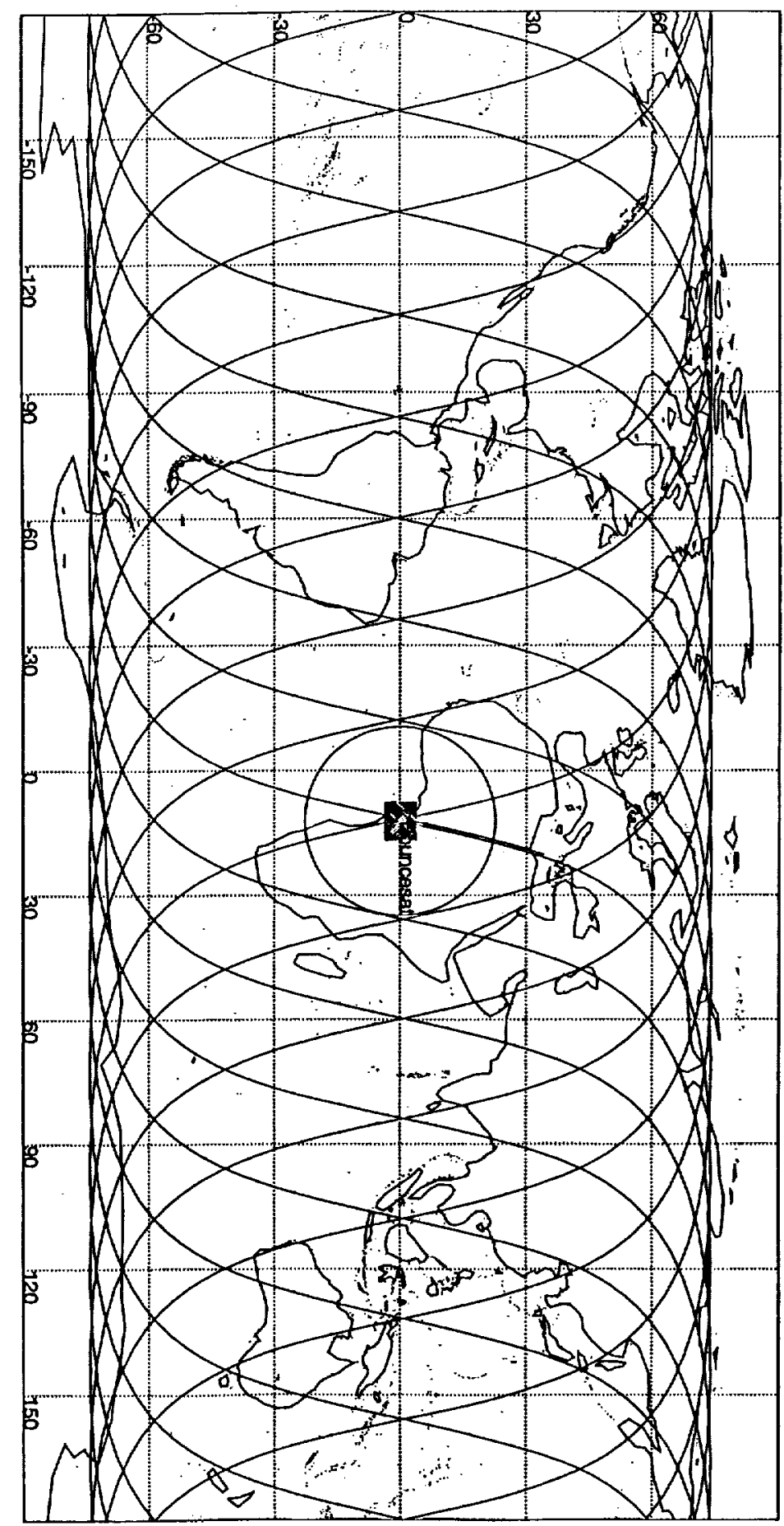

Figure 9 - Scatterometry Mission Groundtracks 


\section{DISCUSSION}

The GPS bounce technology is an exciting new field for geophysical research. Several types of analyses were performed concerning this new type of instrument. First an estimate of the resolution was derived in order to allow comparison with other remote sensing technologies. Then a viewing angle study was completed in order to validate a test flight on the space shuttle. Finally a constellation design analysis was completed to begin mission planning for a future remote sensing mission using GPS reflections.

Resolution was estimated using the size of the glistening zone as a percentage of the satellite's field of view, looking down at nadir. The average number of reflected GPS signals seen at any one time during the simulation was nine. This indicates a little under $10 \%$ of the field of view area was sampled. The distribution of specular points was assumed random.

The viewing angle study saw a uniform distribution of azimuth angles about the down-looking antenna. The elevation study showed higher elevation angles to be more likely. Elevation angles were measured from nadir for this analysis. These results indicate a spaceborne reflection receiver's antenna gain pattern should be designed to maximize high elevation angle signal gain.

A four-satellite constellation has been proposed to enable the realtime mapping of the ionosphere for use in realtime remote sensing corrections and in GPS WAAS. This four-satellite implementation need not be prohibitively expensive. A Pegasus rocket is currently the smallest available launch vehicle. Such a rocket could loft the suggested constellation in two launches. One launch would be for the high inclination satellites, the second for the low satellites. Based on the orbital inclination, the specified altitude, and the known launch vehicle capability, a limit for the spacecraft mass can be obtained. This limit is about $350 \mathrm{~kg}$ per spacecraft (from Loftus \& Texeira ${ }^{23}$ ). This is certainly feasible in terms of today's technology. The scatterometry (or altimetry) mission could either be launched with a single rocket or be flown onboard a future scatterometer (or altimeter). This may be the most useful means of deployment for a GPS reflection receiver. In this way it could provide both backup measurements for the scatterometry mission and provide instantaneous ionospheric corrections. Using a reflection GPS receiver could mean that future altimetry missions do not need to be dual frequency, which may allow cost (and weight) savings on the altimeter itself.

In conclusion, evidence has been presented that GPS reflection receivers could perform several remote sensing missions from low Earth orbit. Work was begun on determining the spatial resolution of a reflection receiver. Coverage studies indicate this instrument is a feasible means of providing scatterometry and ionospheric mapping. It is also recommended that more study be done on carrying a reflection receiver on future altimetry and scatterometry missions. 


\section{ACKNOWLEDGEMENT}

D. Mickler wishes to acknowledge the support of the Colorado Center for Astrodynamics Research during the time this research was conducted.

\section{REFERENCES}

'M. Martin-Neira, "A passive reflectrometry and interferometry system (PARIS): application to ocean altimetry." ESA Journal, Vol. 17, No. 4, pp 331-355, 1993.

${ }^{2}$ J.C. Auber, A. Bibaut, and J.M. Rigal, "Characterization of multipath on land and sea at GPS

frequencies." Proc. OfION GPS-94, Salt Lake City, UT, Inst. of Nav., VA, pp 1155-1171, 1994.

${ }^{3}$ S.J. Katzberg and J.L. Garrison, "Utilizing GPS to determine ionospheric delay over the ocean."

Technical Memorandum, TM-4750, NASA Langley Research Center, Hampton, VA, 13pp, 1996.

${ }^{4}$ J.L.Garrison, S.J. Katzberg, and M.I.Hill, "Effect of sea roughness on bistatically scattered range coded signals from the Global Positioning System." Geophysical Res. Letters, Vol. 25, No. 13, pp 2257-2260, 1998.

5 J.L. Garrison, S.J. Katzberg, M.I.Hill, and C.T. Howell, "Sea State Sensing." GPS World Application Contest VI, GPS World Showcase Issue, August 1998.

${ }^{6}$ A. Komjathy, J.L. Garrison, and V. Zavarotny, "GPS: a new tool for ocean science." GPS World, Vol. 10 No. 4, April, pp 50-56, 1999.

${ }^{7}$ A. Komjathy, "Global Ionospheric Total Electron Content Mapping Using the Global Positioning System," Ph.D. dissertation, Department of Geodesy and Geomatics Engineering Technical Report No. 188, University of New Brunswick, Fredericton, New Brunswick, Canada, 248 pp, 1997.

${ }^{8}$ J.R. Wertz, "Orbit and Constellation Design," in Space Mission Analysis and Design, $2^{\text {nd }}$ ed., Larson \& Wertz, eds., Microcosm Inc., Torrance CA, pp 160-174, 1992.

${ }^{9}$ D.A. Vallado, Fundamentals of Astrodynamics and Applications, McGraw-Hill, New York, pp 294-298, 1997.

${ }^{10}$ L.G. Vargo, "Orbital patterns for satellite systems." Adv. In the Astronautical Sciences, Vol. 6, The Macmillan Company, New York, pp 709-725, 1960.

"J.G. Walker, "Coverage predictions and selection criteria for satellite constellations," Royal Aircraft Establishment Technical Report 82116, Dec. 1982.

12 J.E. Draim, "A six satellite continuous global double coverage constellation," AAS Paper 87-497, AAS/ALAA Astrodyn. Spec. Conf., Kalispell, MN, Aug. 10-13, 1987.

${ }_{13}$ J.E. Draim, "Three- and four-satellite continuous coverage constellations," J. Guid., Control, and Dyn., Vol. 6, pp 725-730, Nov-Dec 1985 .

${ }^{14}$ T.J. Lang, "Optimal low Earth orbit constellations for continuous global coverage," AAS Paper 93-597, AAS/ALAA Astrodyn. Spec. Conf., Victoria, BC, Canada, Aug. 16-19, 1993.

${ }^{15}$ T.J. Lang, "Symmetric circular orbit satellite constellations for continuous global coverage," AAS Paper 87-499, AAS/ALAA Astrodyn. Spec. Conf., in Prog. in Astron. Sci., Vol. 65, Kalispell, MN, Aug 10-13, 1987.

${ }^{16}$ A.H. Ballard, "Rosette constellations of earth satellites," IEEE Trans. On Aerosp. And Elec. Sys., Vol. AES-16, No. 5, pp 656-673, Sept. 1980.

${ }^{17} \mathrm{~L}$. Rider, "Analytic design of satellite constellations for zonal Earth coverage using inclined circular orbits," J. Astron. Sci., Vol. 34, No. 1, pp 31-64, Jan.-Mar. 1986.

${ }_{18}^{18}$ T.J. Lang and J.M. Hanson, "Orbital constellations which minimize revisit time," AAS Paper 83-402, Prog. in the Astron. Sci., Vol. 54, pp 1071-1086, 1983.

${ }_{19}$ J.M. Hanson and A.N. Linden, "Improved low-altitude constellation design methods," J. Guid., controls, and Dyn., Vol. 12, pp 228-236, Mar.-Apr. 1989.

${ }^{20}$ J.M. Hanson, M.J. Evans, and R.E. Turner, "Designing good partial coverage satellite constellations," AIAA Paper 90-2901-CP, ALAA/AAS Astrodynamics Conference, Portland, OR, Aug. 20-22, 1990. 
${ }^{21}$ S.-C. Wu, T. Meehan, and L. Young, "The potential use of GPS signals as ocean altimetry observables," ION 1997 Tech. Mtg., Santa Monica, CA, Jan. 1997.

${ }^{22} \mathrm{M}$. Armatys, personal communication, 1998.

${ }^{23}$ J.P. Loftus, Jr., and C. Texeira, "Launch Systems," in Space Mission Analysis and Design, $2^{\text {nd }}$ ed., Larson \& Wertz, eds., Microcosm Inc., Torrance CA, pp. 669-681, 1992. 